\title{
Mamdani-Fuzzy Framework for Academic Staff Selection and Placement in Nigerian Universities
}

\author{
I.Manga $^{1 *}$, S.D. Samaila ${ }^{2,}$ and H. Bello ${ }^{3}$ \\ ${ }^{1,2,3}$ Department of Computer Science. Adamawa State University Mubi, Nigeria.
}

\begin{abstract}
:
Selection and placement of appropriate personnel for the right job leads to great success in any organization. However, this is one of the most important activities carried out by Human Resource (HR). Minimizing imprecision and subjective value judgment in personnel selection processes were taken into consideration in this research by developing personnel selection and placement framework using the Mamdani- fuzzy model. This research work is aimed at developing Mamdani- fuzzy framework for academic staff selection and placement. A model with three levels has been developed to manage the database, and the necessary conditions required from applicants for selection and placement, and the consideration of individual temperament was paramount. Tools: Java script and HTML (for the front end) PHP and MySQL (for the database storage as back end). Experimental results using fuzzy classification membership function defined by the truth value of a fuzzy propositional function would also be used as part of the analysis and design. When the need arise, MathLab would be employed for some analysis and simulations. A graphical user interface (GUI) would be developed for all the relevant forms in order to effectively interact with the users of the system.
\end{abstract}

Key words: Human Resources, Fuzzy Logic,

\section{Introduction}

Any organization requires resources, including qualified personnel, for effective performance. Researchers in the field of Human Resources Management (HRM) believe that the quality of personnel is a strategic solution because it affects the organization performance, such as, customer satisfaction, innovations, profitability and competitiveness of the company.

Human resources management refers generally to the identification, assessment, selection, employment, motivation, training and development of employees to the goals of the enterprise. It should be also noted that organizations faced the growing global competition in human resources [6].

The personnel selection processes are targeted at selecting and placing the best applicant out of vast pool of applicant to occupy the vacant position identified by HR. A number of techniques which aid organizations in making decisions on how personnel are selected and place properly in suitable positions were adopted.

However, multi criteria decision making (MCDM) techniques have been adopted for handling personnel selection problems [5]. Human resources management refers generally to the identification, assessment, selection, employment, motivation, training and development of employees to the goals of the enterprise. It should be also noted that organizations faced the growing global competition in human resources [6]. The personnel selection process is aimed at choosing the best applicant to occupy vacant positions.

The differences in the expert opinions have caused high degrees of uncertainties in personnel selection..

However, Multi Criteria Decision Making (MCDM) method, Analytic Hierarchy Process (AHP), Technique for Order Preference by Similarity to Ideal Solutions (TOPSIS), Expert Systems (ES), Artificial Neural Networks (ANN), Fuzzy Logic (FL) etc are implemented in applicant selection processes [1].

Much research has been conducted on recruitment policy adaptation due to its critical role in bringing human capital into organizations [9]

Mamdani fuzzy inference is the most commonly seen fuzzy methodology and was among the first control systems built using fuzzy set theory. It was proposed in 1975 by Ebrahim Mamdani [1] as an 
attempt to control a steam engine and boiler combination by synthesizing a set of linguistic control rules obtained from experienced human operators. Mamdani's effort was based on Lotfi Zadeh's 1973 paper on fuzzy algorithms for complex systems and decision processes [2].Fuzzy Logic was adopted as a better method for sorting and managing information and has proven to be an excellent choice for so many Control system applications since it imitates human control logic. It can be built into anything from small, handheld products to large computerized process control systems. It uses an imprecise but very descriptive language to deal with input data more like a human operator. Fuzzy logic system is able to simultaneously handle numeric values and linguistic variable.

Mamdani fuzzy model method is employed in selecting the best applicants by comparing the job specifications with applicant CV so as to rank applicants according to suitability for selection. The analysis is performed by the criteria and fuzzy weighting that can be changed, based on organizational requirements. This framework is very advantageous and thus solves existing problems when implemented in any organization by the HR department.

\section{Related Work}

Fuzzy Logic (FL) was a concept introduced by Zadeh in 1965; a professor at the University of California. The idea of fuzzy logic was to aid in the processing data with ambiguous insinuations. This is achieved by constructing membership functions used for pair wise comparisons. FL offers a simple way of arriving at a logical conclusion in the midst of vague, ambiguous, imprecise, noisy, or missing information. Therefore, fuzzy logic approach to solving computational problems is quite reliable because it replicates how a human would take decisions in a much faster way.

The advantages of using fuzzy logic are enumerated below:

a. It is robust, because it is not only based on precise, unambiguous or noise-free inputs.

b.It can easily be adjusted to enhance or rapidly alter system behavior.

c. It processes a considerable number of inputs and generates the corresponding output based on defined rules.
d.It can control nonlinear systems that would be difficult or impossible to model mathematically. However, using fuzzy logic consists of the following concepts:

i. Defining the modeling aim and parameters: What is to be modeled? How are the parameters injected in order to achieve the aim? What type of output or result is envisioned? What are the like factors that could thwart system success?

ii. Determining the $\mathrm{I} / \mathrm{O}$ relationships and choosing the required number of parameters as input to the fuzzy logic engine.

iii. Using the rule-based instructions to describe the identified problem into series of IF-THEN rules that represent the required output for given input conditions.

iv. Constructing membership functions that defines the parameters of $\mathrm{I} / \mathrm{O}$ values used in the rules.

v. Creating the required pre- and postprocessing routines or programming the rules into the FL engine.

vi. Running the system for performance evaluation in order to ascertain expected results.

To complement the performance of fuzzy logic models, Zadeh in 1973 proposed the concept of linguistic or fuzzy variables which are represented as words or objects instead of numbers. They could be considered as adjectives that represents digital variables. Fuzzy logic models can be categorized into three classes; Mamdani-type fuzzy models, Singleton fuzzy models and TakagiSugeno fuzzy models (Hellendoorn and Drainkov, 1997). The Mamdani fuzzy model is the oldest and its' rule base consist of proficient knowledge and experiences that is capable of predicting knowledge and human inference. On the other hand, Singleton fuzzy model have the feature of predicting system behaviour based on fuzzy rules from I/O data but Takagi-Sugeno models are not single value selection; that is, the antecedent rules are fuzzy variables that output a linear function (Zhang et al., 2010). This research utilizes the Singleton fuzzy model for building the architecture model.

Fuzzy logic is a superset of the conventional Boolean logic with capability for handling 
imprecise (vague) and incomplete data that are commonly found in medical records. It resembles human decision making with its ability to work from approximate reasoning and ultimately find a precise solution to a given problem.

Expert system adopts the concepts of fuzzy logic instead of the Boolean logic to reason about data as its inference mechanism (Aly and Vrana, 2006). It consists of fuzzification, inference, knowledge base, and defuzzification subsystems, and uses a collection of fuzzy MFs and fuzzy rules. It has the capability to solve decision making problems for which no exact algorithm exists by relying on human-like models of approximate reasoning that are expressed in form of fuzzy IF-THEN rules. FES $\mathrm{s}$ well suited to problems that exhibit uncertainty resulting from inexactness, vagueness or subjectivity. The structure of a FES is illustrated in figure 2.1.

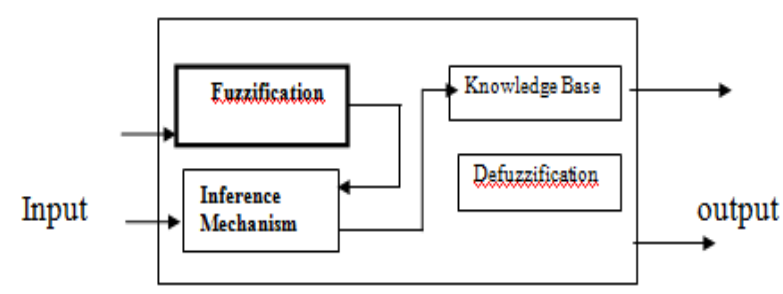

Source: Kosko (1992)

Fig. 2.1 Fuzzy expert system (FES) for personnel selection.

\section{- Knowledge Base}

Generally, this is the component that contains the extracted human expert knowledge in a computer understandable form. In this research, the knowledge that is represented in this component is gotten from four different medical experts who are good at providing medical diagnosis for malaria in a malaria endemic environment. This component is made up of two sub components: Rule base and Database.

Fuzzy rule base: This contains the set of fuzzy IF-THEN rules that embodies the knowledge used by the fuzzy inference system. It represents the knowledge base of the expert system. Each of the rules has an antecedent (IF) and a Conclusion (THEN) part that prescribes what should be done when certain Conditions are true. The specific criteria for selection for each specific job Position are represented as IF-THEN rules which determine applicant suitability
FIS is very popular in computing framework based on the concept of fuzzy set theory, fuzzy IF-THEN ru2es and fuzzy reasoning. The fuzzy inference engine is responsible for evaluating fuzzy rules to produce an output for each rule (Padhy, 2005). The basic structure of fuzzy inference system consists of three conceptual components: a rule base, which contains a selection of fuzzy rules. A database (or dictionary), which defines the Membership Functions (MF) used in the fuzzy rules; and a reasoning mechanism, which perform the inference procedure upon the rules, and the given facts, to derive a reasonable output or conclusion. A fuzzy inference system can take either fuzzy inputs or crisp inputs but its outputs are almost always fuzzy sets. Sometimes it is necessary to have a crisp output, especially in a situation where a fuzzy inference system is used as a controller. Mamdani fuzzy model, Tagaki-Sugeno fuzzy model, and Tsukamoto model are the three most familiar fuzzy inference systems.

\section{- Membership Function (Mf)}

Is a function that specifies the degree to which a given input belongs to a set. A fuzzy set is completely characterized by its MF, and as such the classes of parameterized functions commonly used to define MFs of one dimension are: triangular MF, trapezoidal MF, Gaussian MF, generalized bell MF (Padhy, 2005)

a. Triangular MFs: This is specified by three parameters $(a, b, c)$ where $(a<b<c)$. It can be represented in the form:

$$
\begin{aligned}
& \text { Triangle }(x: \quad a, \quad b, \quad c)=\operatorname{Max}\left[\operatorname { m i n } \left[\frac{x-a}{b-a}\right.\right. \\
& \left.\left.\frac{c-x}{c-b}\right] 0\right](1)
\end{aligned}
$$

b. Trapezoidal MFs: A Trapezoidal MFs is specified by four parameters $\{a, b, c, d\}$

Where $(a<b<=c<\mathrm{d})$.

Trapezoidal $(x: a, b, c, d)=\operatorname{Max}\left[\min \left[\frac{x-a}{b-a}\right.\right.$,

$\left.\left.1, \frac{d-x}{d-c}\right] 0\right]$

(2)

c. Gaussian MF: A Gaussian MF is specified by two parameters $\{c, \sigma\}$; where $c$ is the MF's centre and $\sigma$ determines the MF's width. 
$\operatorname{Gaussian}(\mathrm{x}: \quad \mathrm{c}, \quad \sigma)=\exp \left[\frac{-(x-c)^{2} / \sigma^{2}}{2}\right]$ (3)

d. Generalized bell MFs: A generalized bell MF also called bell MF is defined by three parameters $\{a, b, c\}$ :

A. Bell $(x a b c)=A$. $\frac{1}{1+\frac{x-c}{a}}$

Where parameters $a$, represents the width, c represents the MF's centre and $b$ represents the slope of the crossover point. It is usually positive (if $b$ is negative, the shape of this MF becomes an upside-down bell).

\section{- Fuzzy Logic Basic Operators}

The basic operations that could be performed on fuzzy sets include:

\section{- The Compliment}

Complement operations is mostly performed on fuzzy sets that shows to what extent an element does not belong to the set. For instance, if we have the set of tall women, its complement is the set of NOT tall women. The membership representation is shown in Figure 2.3.

Figure 2.3 Compliment of fuzzy set
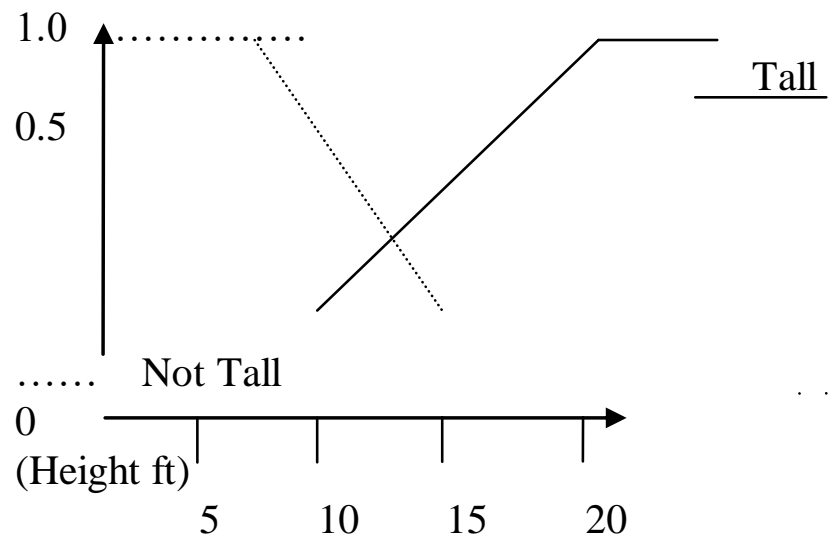

Fig. 2.1 Membership Grade.

If $A$ is the fuzzy set, in a universe $X$. Its complement $A$ can be found as follows:

$$
\mu A(x)=1-\mu A(x) \text { for all } x \in X
$$

\section{- The Intersection Operator}

This shows the extent to which an element is in both sets as shown in Figure 2.1. For example, the intersection of the 3 set of tall men and the set of fat men is the area where these sets overlap. A fuzzy intersection is the lower membership in both sets of each element. The fuzzy intersection of two fuzzy sets $\mathrm{A}$ and $\mathrm{B}$ on universe of discourse $X$ :

$\mu A \cap B(x)=\min [\mu A(x), \mu B(x)]=\mu A(x) \cap \mu$ $B(x)$ for all $x \in X$

Figure 2.

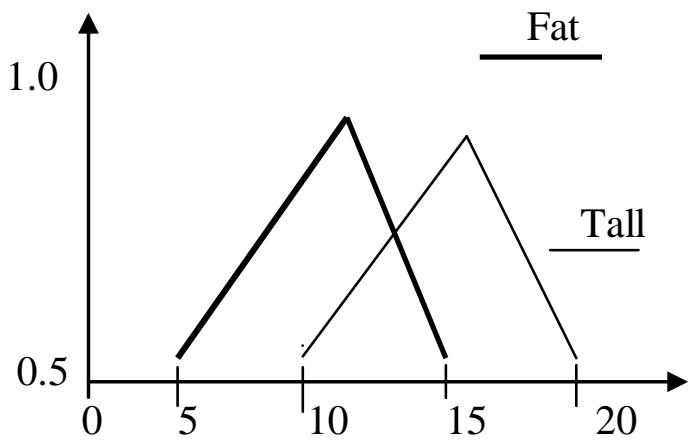

Fig. 2.2 Intersection of fuzzy sets Membership Level $\mathrm{F} \cap \mathrm{T}$

\section{- The Union Operator}

This shows the number of the element in the set. In fuzzy sets, the union is the reverse of the intersection. That is, the union is the highest membership value of the element in either set as depicted in Figure 2.3. For instance, the union of tall men and fat men contains all men who are tall OR fat. The fuzzy operation for forming the union of two fuzzy sets A and B on universe $\mathrm{X}$ can be obtain as:

$\mu A \cup B(x)=\max [\mu A(x), \mu B(x)]=\mu A(x) \cup \mu$ $B(x)$, for all $x \in X$ (2.3)

\section{Research Methodology}

Mamdani- fuzzy model is the method used for this research which is most widely used fuzzy inference systems and the data collected for the system testing is obtained from experts and the analysis of present relevant documents on the application of fuzzy logic for personnel selection. Fuzzy scores is calculated for each alternative by multiplying the scaled value given to the alternative of that attribute with the weights of relative importance directly assigned by decision maker followed by adding the products of all attributes. The advantage of this method is that the relative order of importance of the standardized scores remains equal. 
The process of academic staff selection using the Mamdani fuzzy involves the following stages:

i. Fuzzification of input variables (values of signs, symptoms, and laboratory test results).

ii. Establishment of the fuzzy rule base.

iii. Developing the decision making logic of the fuzzy logic component (inference engine).

iv. Defuzzification of the output of the inference engine into crisp values.

In Modeling the Mamdani fuzzy system for academic staff selection, seven (7) different input parameters were used to evaluate applicant suitability for the job based on the recommendation that academic staff selection processes can be carried out using applicant qualification, Applicant temperaments, class of degree, gender, age, years of working experience, and the course of study.

Fuzzy weights are assigned to each parameter in order of importance to the organization.

After matching the job specifications with applicant CV, the system, automatically shortlist and notify applicants to proceed to the next level of selection processes. The scores obtained by applicants are evaluated in the fuzzy engine for the final ranking.

The following algorithms were adopted in this research work:

i. Human Resource Manager sets all requirements for the available job(s)

ii. The available vacancies are advertised.

iii. The applicants apply for the job vacancy.

iv. Applicants are short listed and notify via email and SMS when match is found.

v. Shortlisted Applicants are interviewed by the interview panels,

vi. Applicant scores are evaluated and ranked based on suitability check using Mamdani fuzzy model.

vii. Applicants are accepted or rejected depending on the fuzzy scores obtained.

A case study to demonstrate how the algorithm works is presented.

\section{- An Illustration of the Applicant Selection Problem}

The relevance and the applicability of the FES for personnel

Selection can be demonstrated using an illustration whereby University wishes to select two applicants for various job positions and wishes to evaluate their submitted $\mathrm{CV}$ in respect of the specific job advertised whereby the job requirements such as: Applicants temperament, Qualification, age, gender, work experience, certifications, and course of study are clearly specified. We assume that two academic positions are considered to be vacant in computer Science department and required to be filled with the requirements stated as follows in Table 1.

Table 3.1 Academic staff selection requirements

\begin{tabular}{|l|l|l|}
\hline S/n & Criteria & Fuzzy values \\
\hline 1 & Qualification & Msc. \\
\hline 2 & Class of Degree & $\begin{array}{l}\text { Minimum (second Class } \\
\text { Lower) }\end{array}$ \\
\hline 3 & $\begin{array}{l}\text { Academic } \\
\text { Experience }\end{array}$ & Academic Experience \\
\hline 4 & Course of Study & $\begin{array}{l}\text { Computer } \\
\text { (preferably) }\end{array}$ \\
\hline 5 & Gender & Male (preferably) \\
\hline 6 & Age & Age range of 25-40 \\
\hline 7 & Temperament & Sanguine \\
\hline
\end{tabular}

In order to overcome the weaknesses identified in the literatures reviewed, this research paper places much emphasis on the fairness and drastically minimizes human subjectivity. To achieve this, Fuzzy weights are assigned to each parameter which is important in the Staff selection processes depending on the job descriptions. This flexibility of assigning scores to the membership function is the hallmark of the research. From the algorithm the parameters obtained from the requisition are as follows: Qualifications, Course of Study, Class of Degree, Temperament, Academic experience age, and gender and work experience. These parameters are used in order to short list applicants. All short listed applicants write aptitude test, computer based test and temperament test. Since this research is basing the scoring on multiple attributes, the figures below illustrate the multiple attribute decision that needs to make in order to achieve the objective. Here different kinds of weights are assigned to the parameters in order of importance or ratings. The fuzzy weights when summed up should be less or equal to 1.0. In order to obtain the fuzzy score for the applicants, so that they can be ranked.

The attributes in figure 3.1 are assigned with fuzzy points each attribute is broken down into sub sections and the allocated Fuzzy weights. These weights are either equal to 1.0 or less than 1.0. The corresponding fuzzy points under each 
category sum up to the weight assigned to the attribute in general.

The range of input values for selection per requirement is given as follows:

\section{Qualification (0,2)}

i. Msc, Bsc/HND. Computer Engineering $/ 0.02$

ii. Msc, Bsc/HND. Computer Science/0.04

iii. Phd,Msc, Bsc/HND. Operational Research/0.06

iv. Phd,Msc, Bsc/HND, Computer Science $/ 0.08$

The minimum class of degree $2.5<x \leq 5.0$ (maximum grade point 5.0)

\section{Work experience $(0.1)$}

I. $0-4$ years $/ 0.02$

II. $5-6$ years $/ 0.03$

III. $7-8$ years $/ 0.05$

The minimum working experience is $4<x \leq 8$

year

Temperament $(0.1)$

i. Suitable/0.06

ii. Non suitable/0.04

\section{Gender (0.1)}

i. Female/0.06

ii. Male/0.04

Age (0.1)

i. 24 - 39 years $/ 0.06$

ii. $40-65$ years $/ 0.04$

The minimum age of $24 \leq x \leq 60$ years

\section{Class of degree (0.1)}

i. First Class (1-1)/0.05

ii. Second class Upper (2-1)/0.03

iii. Second class Lower (2-2)/0.02

\section{Course of Study $(0.1) \mathrm{v}$}

i. Computer science/0.05

ii. Computer Engineering/0.03

iii. Operational Research/0.02

\section{- Experimental Evaluation}

In solving this problem, fuzzy weights have been assigned to different types parameters in order of importance to the specific job requirement in the interval of either equal to 1.0 or less than 1.0. As an example, the case of four (4) candidates that have applied for the post of senior lecturer with differing credentials is shown in Table 2.

Table 3.2: personnel information

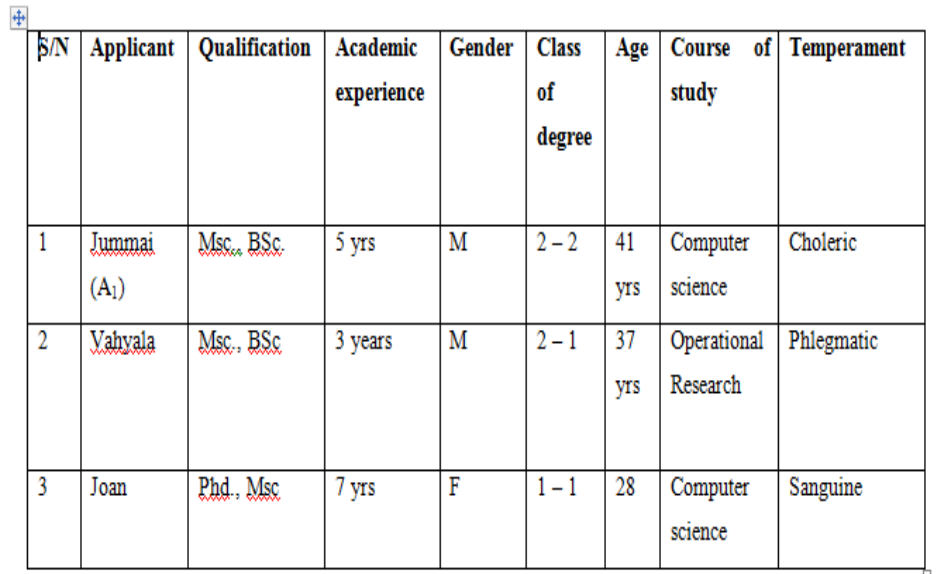

Source: (Field work, 2018)

The fuzzy set representation for the six (6) basic criteria is represented as:

Qualification: (PhD. Msc. Bsc./HND, Computer Science $) / 0.08+$ (PhD. Msc. Bsc/HND. Operational Research) $/ 0.06+$ (Msc. Bsc./HND. Computer Science) $/ 0.04+\left(\mathrm{MSc}^{\prime}+\right.$ PGD*)/0.6 + (Msc. Bsc/HND. Computer Engineering)/0.4 Course of study:

Class of degree: $1.0 / 0+y=0.02+x / 0.03+$ $0.05 / 1$ (using the linear MF)

YOE: $5 / 0+\mathrm{y}=-0.67+x / 4+x / 5+10 / 1$

Age: $28 / 0.06+y=37 / 0.06+41 / 0.04+52 / 0.04$ (using triangular MF).

Using triangular MF to evaluate the fuzzy scores of Age parameter:

$25 / 0+26 / 0.33+27 / 0.67+28 / 1+29 / 0.5+30 / 0$

Then, the membership values for the requirement parameters of the first, second, and third Applicants are given as follows:

$\mathrm{A}_{1}=1 / 0.02+2 / 0.03+3 / 0.04+4 / 0.02+5 /$ $0.04+6 / 0.05+7 / 0.04$

$A_{2}=1 / 0.02+2 / 0.02+3 / 0.04+4 / 0.03+5 /$ $0.06+6 / 0.02+7 / 0.04$

$\mathrm{A}_{3}=1 / 0.05+2 / 0.06+3 / 0.05+4 / 0.06+5 / 0.06+$ $6 / 0.05+7 / 0.08$

The fuzzy hamming distance measure for the three applicants considered using all the seven parameters as shown in Table 3.3

The final fuzzy score obtained by each applicant is shown in the table 3.3. If the job vacancy for only one person to occupy, it is clear that Applicant $\mathrm{A}_{3}$, should be placed based on the ranks obtained. The research work focused mainly on the evaluation of the results based on the Mamdani Fuzzy Model. Finally, the applicant $\mathrm{A}_{3}$ having scored 0.146 is best applicant to fit the job. 
This computationally evaluates the closeness of each applicant fuzzified credentials to the job requirements for a specific job position using the fuzzy hamming distance function.

\section{RESUlTS, DISCUSSION AND CONCLUSION}

After collating and evaluating all applicant records, the Mamdani Fuzzy framework produces Table 3.2 where three applicants were short listed: Jummai $\left(A_{1}\right)$, Vahyalla $\left(A_{2}\right)$, and Joan $\left(A_{3}\right)$. As shown in Table 3.3, applicant $\mathrm{A}_{2}$ has the largest score due to the most appropriate choice. Finally, and upon the results of experiments, it is clear that based on the Mamdani Fuzzy approach, the applicant with the highest score is the most suitable choice for the job while the candidate with the smallest score among the three others is ranked in the last place.

\subsubsection{The Login Interface}

Fig.4.1 shows the login interface which opens the main interface when a match is found. All the users of the system utilize this interface as a starting point to using the fuzzy personnel selection system.

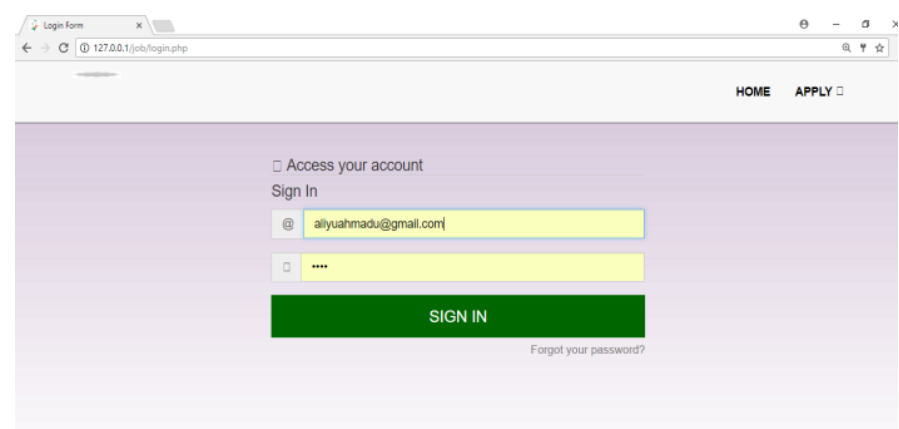

Fig.4.1 the login interface

\section{- Applicant Dash board}

Figure 4.2 shows the main user interface and main menu of the personnel selection system using FSAW where depending on the level of applicants in the selection processes, only the specific aspects are displayed to them. The System menu allows the applicants to carry out various activities: submit application, and write test.
WELCOMEI YOU CAN PROCEED YOUR APPLICATION ACTIVTIES HERE

Fig.4.2 the Applicant Dashboard

\section{- Open and Close vacancy}

Figure 4.0 describes the dialog box used for opening and closing vacancy period. Applicant's record cannot be inputted into the system. From the research, it was realized that the system administrator could open the period for the process and close it within a period of time.

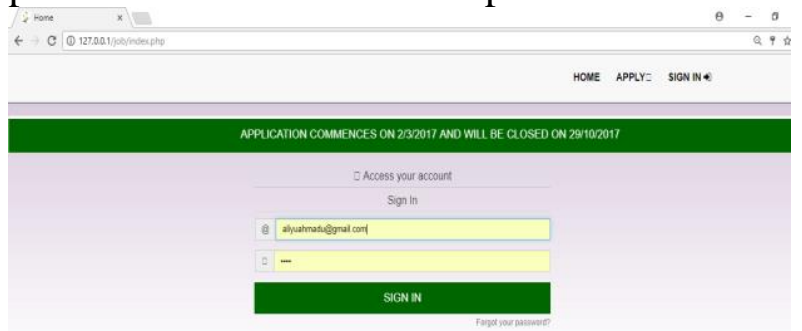

Fig. 4.3 Open and Close vacancy

\section{- Applicant Record}

Figure 4.1 is used to enter the data obtained from the applicants. When the applicant's ID is selected, the name populates in the text field and the attributes are then set.

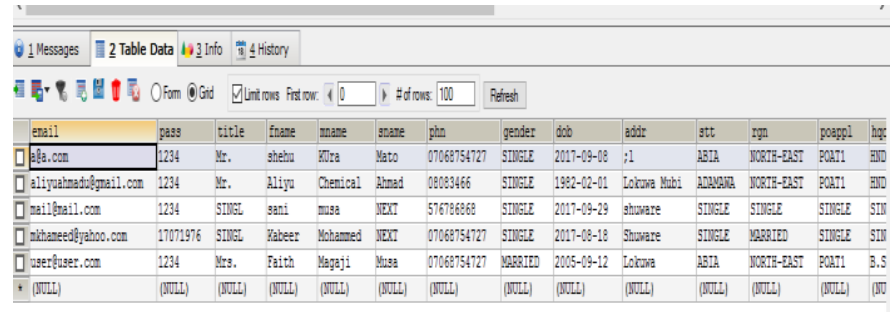

Fig.4.4Applicants untreated reports 


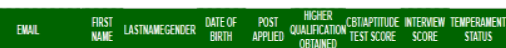

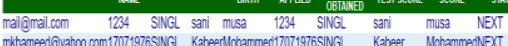

\subsection{CBT}

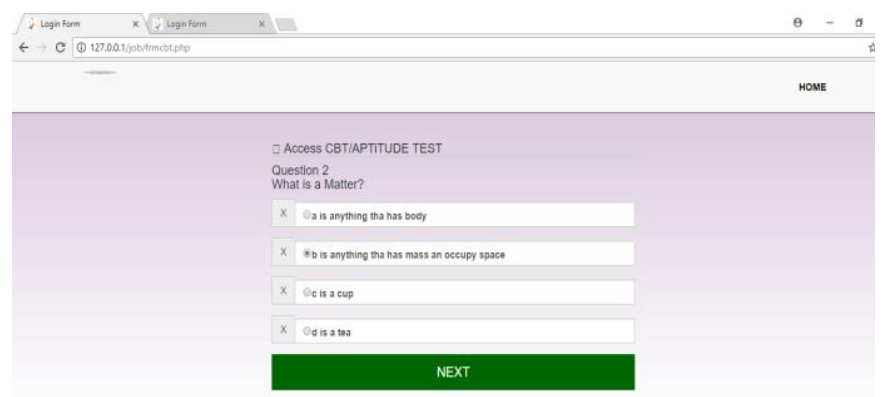

Fig. 4.6 CBT and temperament Test

\section{- Applicant Interview}

Where applicants are being interviewed based on three factors: confidence level, composure, and the language spoken.

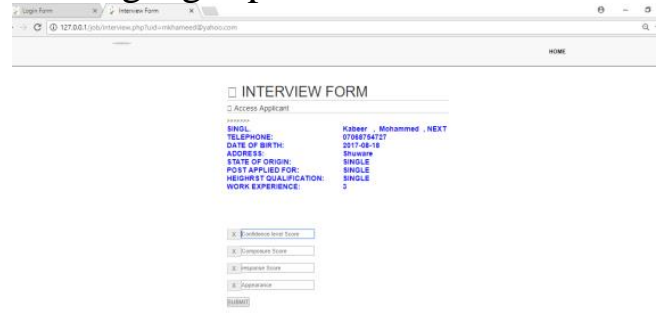

\section{- Qualified candidates Screening Form}

\section{SUSSEFUYY EMPLOYED CANOIDATES CANDDDATES}

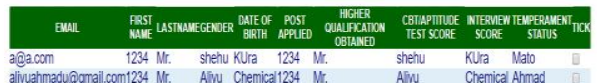

Fig. 4.7Qualified candidates Screening Form

\section{Conclusions}

Personnel Selection is one of the major problems that are existing in many organizations for a very long time, which resulted in attracting many researchers' attentions. The reliability of traditional methods such as interviews or applicants test is highly questionable. However, some researchers have focused on the application of Techniques for Order Preference by Similarity to an Ideal Solution (TOPSIS), Fuzzy Elimination Et Choix Transduisant la REaite (ELECTREC), Fuzzy Analytical Hierarchy Process (AHP), Preference Ranking Organization Method for Enrichment Evaluation (PROMETHE), and SAW methods, expert systems, to deal with personnel selection problem.

Mamdani fuzzy framework can be employed in solving decision maker's problem in applicant selection. Data are obtained from expert and the analysis of relevant documents in the field fuzzy Logic for personnel selection for system testing. A new method has been presented for evaluating the overall suitability of job applicants, using the weights allocated to different parameters in advance of applicant evaluation; the fuzzy method achieves a rational basis for assessment. However, It is recommended that an individual's medical examination (medical tests that are conducted to ensure physical fitness of the potential employee/applicant be factored and a fuzzy number assigned to influence applicants chance of obtaining the job. This will greatly decrease chances of employee not coming to work regularly which will affect organizational performances. To further minimizes human subjectivity the system could be developed with the ability of online test, after shortlist applicants could be interviewed and successful applicants are notified email and SMS.

\section{References}

[1] Afshari, A.R., Nikoli, M., and Cockalo, D. (2014). Applications of Fuzzy Decision Making for Personnel Selection Problem a Review. Journal of Engineering Management and Competitiveness, vol.4 issue10,pp 68-77

[2] Aly, S. and Vrana, I. (2006) toward efficient modeling of fuzzy expert systems: a survey.Agriculture Economics, Vol.52 issue10,pp456-460.

[3] Daramola J.O.,Oladipupo O.O and Musa A.G. (2010). A fuzzy expert system (FES) tool for

online personnel recruitments. International Journal Business Information Systems, vol.6 issue 4.

[4] Dursun, M., and Karsak, E. E. (2010). A fuzzy MCDM approach for personnel 
selection. Expert Systems with Applications, vol.37 issue6, pp 4324-4330.

[5] Khorami, M., and Ehsani, R. (2015). Application of Multi Criteria Decision Making Approaches for Personnel Selection Problem: A Survey. International Journal of Engineering Research and Applications, vol.5 issue 5)pp14-29.

[6] Padhy, N.P. (2005) Artificial Intelligence and Intelligent Systems, pp.1-632, Oxford University Press, New Delhi.PetrovicLazarevic.

[7] Samaila V.D., Gumpy G. M., and Manga I (2017). Application of fuzzy logic for personnel Selection. International Journal of Computer Applications (179(15).

[8] Safari, S.M., Vazin, K., \& Ali K, 2014. Identifying and ranking the Human Resources Management Criteria influencing on organizational performance using MADM Fuzzy techniques, Management Science Letters,vol.4, pp.1577-1590.

\section{Authors Profile}

\section{Samaila Vandi Dia}

received the B.Sc. and M.Sc. degrees in Computer Science from Adamawa State

University Mubi, Nigeria in 2010 and 2018, respectively.

\section{Manga Ibrahim}

holds a B.Sc and M.Sc Computer from Adamawa State University, Mubi in 2007 and 2014 respectively. $\mathrm{He}$ is currently undergoing a $\mathrm{Ph} . \mathrm{D}$ programme in Modibbo Adama University of Technology, Yola Nigeria.

His research interest include AI and Data compression. 